\title{
Instability of supersonic ramp flow with intermittent transition to turbulence
}

\author{
Kangping Zhang, Neil D Sandham, and Zhiwei Hu
}

\begin{abstract}
This paper investigates the global instability of supersonic ramp flow at Mach number 4.8 at different Reynolds numbers through direct numerical simulations. It is found that a critical Reynolds number exist for the flow to become globally unstable. Transition to turbulent happens downstream of the ramp with strong intermittency and is due to the breakdown of streaky structures fed by instability from the ramp separation region.
\end{abstract}

\section{Introduction}

Ramp flows are commonly seen in high-speed vehicle control surfaces and engine intakes. Flow separation, transition and shock-wave/boundary-layer interaction (SWBLI) may exist simultaneously in such flows and influence each other. Instability studies with SWBLI have been carried out in the context of flow transition[1]. Balakumar et al.[2] studied the stability of high speed compression corners using both direct numerical simulation (DNS) and linear stability theory, and showed that the added disturbances grew exponentially both upstream and downstream of the separated region but remained neutral across the separation area. Transition to turbulence only occurs downstream, due to second-mode (called the Mack mode) of instability. Ludeke and Sandham [3] studied ramp flow with Mach number 4.8 at different Reynolds numbers. Small secondary recirculation zone was found at a Reynolds number of 3,422 . They observed a convective transition process in a threedimensional (3D) ramp flow. This paper investigates using DNS the instability of ramp flow at Mach number 4.8 at higher ramp angle for different Reynolds numbers and considers the mechanism of transition to turbulence in the presence of global instability.

Kangping Zhang (now at CARDC, China) $\cdot$ Neil D Sandham $\cdot$ Zhiwei Hu AFM Group, Faculty of Engineering and the Environment, University of Southampton, Southampton SO17 1BJ, United Kingdom, e-mail: z . hu@ soton. ac . uk 


\section{Numerical Method and Computational setup}

The simulations presented in this paper solve the unsteady, 3D compressible NavierStokes equations using DNS, non-dimensionally using the inflow freestream velocity $U_{\infty}^{*}$, density $\rho_{\infty}^{*}$, temperature $T_{\infty}^{*}$ and viscosity $\mu_{\infty}^{*}$ are used, together with the inflow boundary layer displacement thickness $\delta_{0}^{*}$ as reference quantities. The dynamic viscosity $\mu$ is assumed to follow the Sutherland's law, with the Sutherland temperature set to $110.4 \mathrm{~K}$. The Reynolds number is defined as $\operatorname{Re}=\rho_{\infty}^{*} u_{\infty}^{*} \delta_{0}^{*} / \mu_{\infty}^{*}$. A constant Prandtl number $\operatorname{Pr}=c_{p}^{*} \mu^{*} / k^{*}$ of 0.72 is used.

A fourth-order central difference scheme is employed to discretize internal points with a stable boundary treatment, which guarantees overall fourth-order spatial accuracy. Time marching is performed using a low-storage third-order explicit compact Runge-Kutta method. The non-linear inviscid flux is split into conservative and non-conservative parts for stability improvement. A TVD scheme with a Ducros sensor, which only switches on the TVD scheme outside the boundary layer, is used for shock capturing. Details can be found in Sandham et al. [4].

Simulations are run at a ramp angle of $12^{\circ}$ with freestream Mach number 4.8 and static temperature 55.4 K. Two cases with Reynolds numbers of 3,422 and 6,843, based on the inflow boundary layer displacement thickness, are simulated. After some tests the computational domain is chosen to extend 215.2 and 325 (in nondimensional unit) before and after the ramp corner, respectively, and has a height of 50 in the wall-normal direction. The domain is meshed with stretched grids in both the streamwise and the wall-normal directions. Mesh convergence study found that a mesh of $1,800 \times 250$ gives grid independent results. Meshes for 3D simulations are extruded from the two-dimensional (2D) mesh with the span equal to one wavelength of the added disturbance, ranging from 3 to 24 . To resolve the flow in the spanwise direction, 16 points are used across the span for the study of the exponential stage of 3D global instability. This is considered to be sufficient to resolve the flow at linear growth stage as only one wave is added in the span. Finer mesh with $N z=64$ is used for for transition study.

Samilarity solutions for laminar boundary layer are applied at the inflow. Characteristics boundary condition is applied at the outflow and top boundaries. The wall is considered to be isothermal with temperature equal to its laminar adiabatic wall temperature. Period boundary condition is used in the spanwise direction.

\section{Results}

Code validation and a grid independence study is first carried out. A 2D case is run for a $6^{\circ}$ compression ramp at $M=4.8$ and $R e=6,843$. Good agreement is obtained with Ludeke and Sandham[3] and Pagella et al. [5]. Simulations are then run in 2D at $12^{\circ}$ at $M=4.8$ and $R e=3,422$ and 6,843. Steady solutions are obtained for both cases, as shown in figure 1 below, with recirculation zones clearly seen in both cases. The separation region is $L_{s}=241$, extending from $x=84$ to 325 for the low Reynolds 

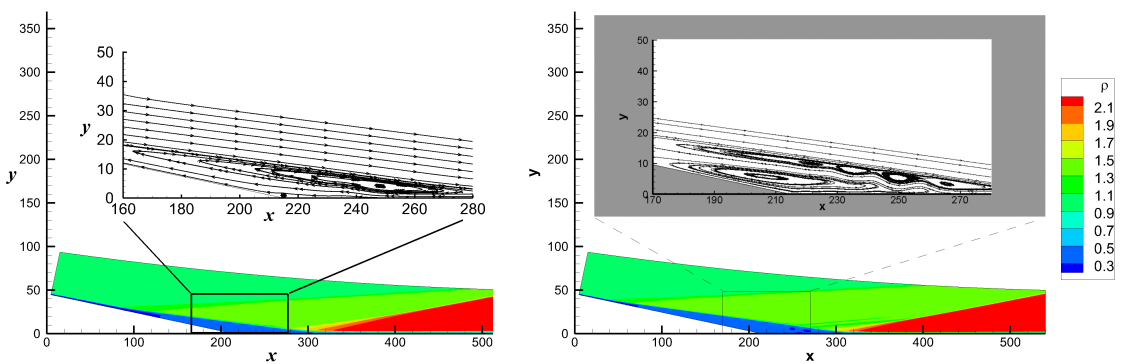

Fig. 1 The separated flow for the $12^{\circ}$ ramp at Reynolds number 3,422 (left) and 6,843 (right).

number case. A larger separation region appears for the higher Reynolds number case with $L_{S}=292$ (from $x=40$ to 332 ). Small secondary recirculation zones can also be found for this case.

The steady 2D solution is used as a base flow for a 3D stability study. The 3D base flow is obtained by extending the $2 \mathrm{D}$ base flow in the spanwise direction. Small disturbances are added to the base flow in the spanwise velocity component to study the global instability. The disturbance is confined within a small patch above the ramp corner in the $x-y$ plane with a Gaussian profile and a single wave along the span, in the form of

$$
w^{\prime}(x, y, z)=A e^{-\frac{1}{4}\left[\left(x-x_{c}\right)^{2}+\left(y-y_{c}\right)^{2}\right]} \sin \left(\frac{2 \pi z}{L_{z}}\right),
$$

$\left(x_{c}, y_{c}\right)=(234.7,2.81)$ is the centre of the Gaussian profile, the amplitude is chosen as $A=3.5 \times 10^{-12}$. Development of flow three-dimensionality is monitored by a statistical measure of the growth of the disturbances via

$$
\varepsilon(x, y)=<w(x, y, z)^{2}>-<w(x, y, z)>^{2},
$$

where $<$. $>$ denotes averaging over span.

Simulations are run for $L_{z}=12$ at both Reynolds numbers. The growth of disturbances at a monitor point upstream of the ramp corner at $(196.76,6.7)$ is shown in figure 2. It can be seen the flow is stable for the low Reynolds number case, however it becomes unstable at high Reynolds number, suggesting there exists a critical Reynolds number for flow instability, with the threshold in between the two Reynolds numbers. Further analysis of the flowfield showed the instability firstly developed at the centre of the secondary recirculation zone. The magnitudes of the maximum reverse flow reaches $19.5 \%$ and $26 \%$ of the freestream speed at the low and high Reynolds number, respectively. So the high Reynolds number flow is likely to be absolutely unstable in three dimensions, while the low Reynolds number case is neutral or might need more time to become unstable. 
Fig. 2 Growth of disturbances $\varepsilon$ with time for $L_{z}=12$ at different Reynolds numbers for a monitor point at $(196.76$,

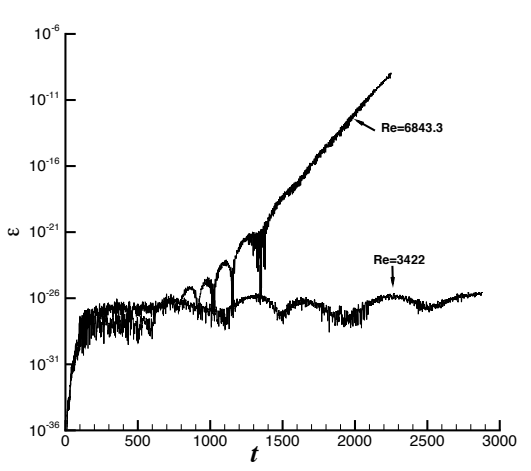
$6.70)$.

The high Reynolds number case is further studied with added disturbances of different spanwise lengths $L_{z}$ of 5, 6, 8, 12 and 24. The growth of disturbances for all cases is illustrated in figure $3 \mathrm{a}$. It is found that the growth rate of disturbances varies with the wavelength of the added perturbation. By measuring the slopes in the exponential stage, variation of the growth rate is shown against wavenumber in figure $3 \mathrm{~b}$. The peak of the growth rate corresponds to the case with $L_{z}=12$. Error bars in the growth rate are calculated from the growth rates for different monitor points. The flow is most unstable with a spanwise length of 12 times the inflow boundary layer displacement thickness.

In the exponential stage of disturbance growth, the development of the added $w$ velocity perturbation still follows the single-spanwise harmonic structure, with the highest amplitudes appearing in the region for $x=230$ to 320 and $y=0$ to 20 , within the flow recirculation zone.
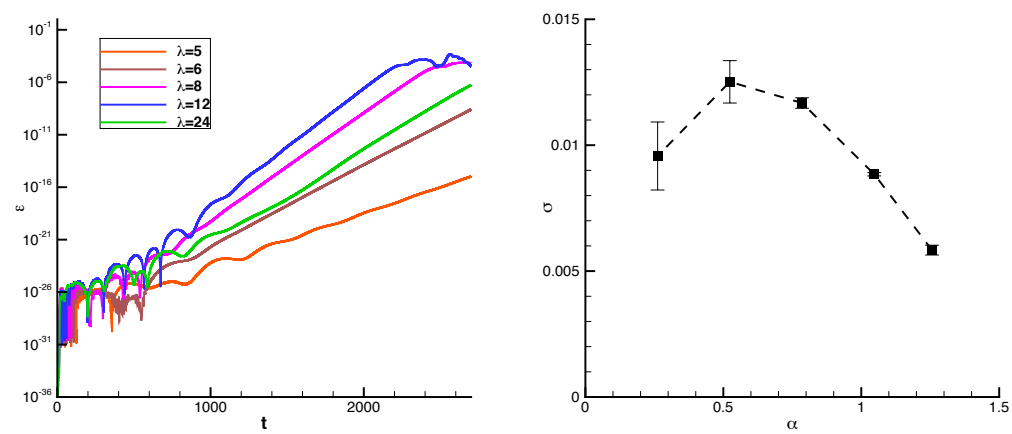

Fig. 3 Statistical measures of instability for ramp flow with different spanwise lengths: (a) variation of $\varepsilon$, defined by equation (2) at monitor point $(246.86,2.81)$ with time, (b) the growth rate against wavenumber, with error bars measured from growth rate at different monitor points. 
Flow transition is further studied for $R e=6,834$ for the most unstable case with $L z=12$. The spanwise grid points are increased to $N_{z}=64$ for improved resolution. Flow structures at different time instances are shown in figure 4 . It can be seen that disturbances start to grow in the recirculation zone near the ramp corner, which lead to the formation of streamwise vortices. Visualization of flow at different streamwise locations (not shown here due to space limitation) shows that the shear flow and reattachment shock push the vortices towards the wall and lead to streaks. As the flow develops the streaks grow in strength. Streak amplitudes of more than $29.5 \%$ of the freestream speed are observed, breakdown to turbulence happens further downstream. This is consistent with existing studies that an streak amplitude of about $26 \%$ was needed for streak breakdown to occur. It is also observed that the turbulence flow is strongly intermittent.

\section{Conclusions}

Direct numerical simulations are performed for supersonic ramp flow at Mach number 4.8 to investigate the instability and transition process. A critical Reynolds number exists, between the two cases study for $R e=3,422$ and 6,843 , for the global instability. For the ramp flow with the high Reynolds number, disturbances with a spanwise wavelength equal to 12 times the incoming boundary layer displacement thickness is found to be most unstable. The disturbances generated by the recirculation zone do not lead directly to transition to turbulence. Instead, streamwise vortices are formed. Because of the shear flow and reattachment shock, the vortices are pushed towards the wall and lead to streamwise velocity streaks. The streaks reach high amplitude and breakdown to turbulence happens further downstream with strong intermittency.

Acknowledgements The computer time is provided by the UK Turbulence Consortium under Engineering and Physical Sciences Research Council (EPSRC, UK) Grant No. EP/G069581/1.

\section{References}

1. Robinet, J.-C. : Bifucations in shock-wave/boundary-layer interaction: global instability approach, J. Fluid Mech., 579, 85-112 (2005).

2. Balakumar, P., Zhao, H. and Atkins, H. : Stability of hypersonic boundary layers over a compression corner, AIAA J., 43, 760-767 (2005).

3. Ludeke, H., and Sandham N.D. : Direct numerical simulation of the transition process in a separated supersonic ramp flow, AIAA Paper, 2010-4470, (2010).

4. Sandham N.D., Li, Q., and Yee, H. C. : Entropy splitting for high-order numerical simulation of compressible turbulence, J Comp. Phys., 178(2), 307-322 (2002).

5. Pagella, A., Babucke, A. and Rist, U. : Two-dimensional numerical investigations of smallamplitude disturbances in a boundary layer at $M a=4.8$ : Compression corner versus imping shock wave, Phys. Fluids, 16(7), 2272-2281 (2004). 

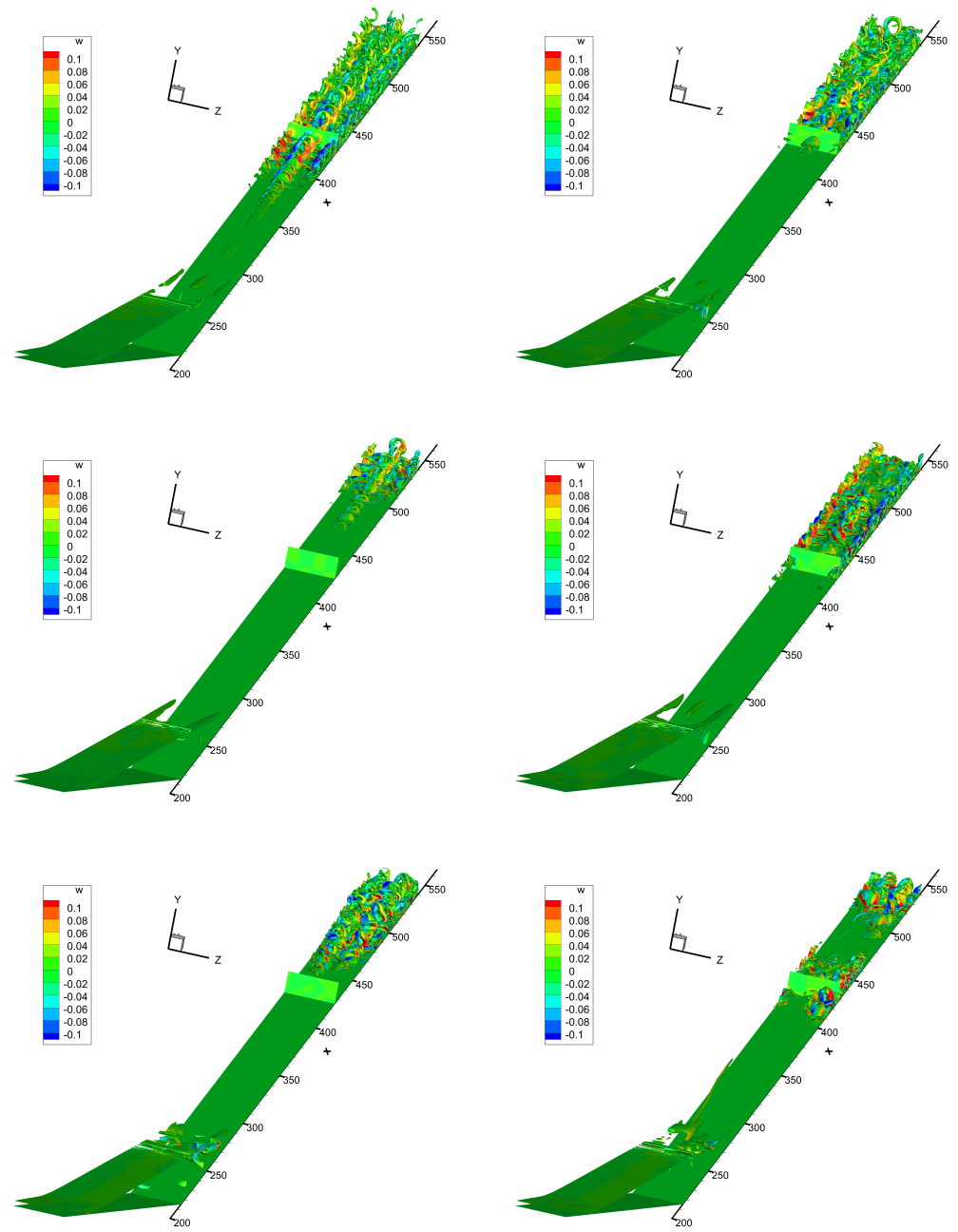

Fig. 4 Iso-surface of the normalized second invariant of the velocity grandient vector at $Q=0.01$, coloured by the spanwise velocity at time instances (from top left to bottom right) $t=2925,3150$, $3375,3600,5400$ and 5513. 